\title{
Mujeres que finalizaron una relación maltratante: características de personalidad, psicopatológicas y sociodemográficas*
}

Women who finished a violent relationship: Personality, psychopathological, and socio-demographic characteristics

Recibido: septiembre 8 de 2010 | Revisado: octubre 25 de 2010 | Aceptado: noviembre 2 de 2010

\author{
Paola Marcela Preciado-Gavidia** \\ NANCY ELIZABETH TORRES-CENDALES \\ CÉSAR ARMando ReY-Anacona \\ Universidad Pedagógica y Tecnológica de Colombia
}

\section{RESUMEN}

Se examinaron las características de personalidad, psicopatológicas y sociodemográficas de mujeres que finalizaron una relación maltratante de pareja $(n=54)$, comparándolas con mujeres que continuaron con este tipo de relación $(n=41)$, todas con edades entre 18 y 60 años $(M=37.4)$. Se utilizó un ficha sociodemográfica, la prueba Big Five Questionnaire (Caprara, Barbaranelli, Borgogni \& Perugini, 1995) y la Mini International Neuropsychiatric Interview (Sheehan \& Lecrubier, 2000). Las participantes que finalizaron la relación presentaron mayores puntuaciones en Afabilidad, Estabilidad Emocional y Apertura Mental, un menor número de hijos, mayor búsqueda de ayuda en instituciones gubernamentales tras los episodios de violencia y una menor prevalencia de episodio depresivo mayor, trastorno distímico, riesgo suicida leve, trastorno de angustia, agorafobia y trastorno de ansiedad generalizada.

Palabras clave autores:

Violencia de pareja, mujeres, personalidad, psicopatología, características demográficas.

Palabras clave descriptores:

Psicología Clínica, Big Five Questionnaire (BFQ), diseño descriptivo.

Para citar este artículo: Preciado-Gavidia, P. M., Torres-Cendales, N. E. \& Rey-Anacona, C. A. (2012). Mujeres que finalizaron una relación maltratante: características de personalidad, psicopatológicas y sociodemográficas. Universitas Psychologica, 11(1), 43-54.

* Esta investigación corresponde a una tesis de grado de psicología realizada por las dos primeras autoras, bajo la dirección del tercer autor.

** Paola Marcela Preciado Gavidia: Psicóloga Universidad Pedagógica y Tecnológica de Colombia. Nancy Elizabeth Torres Cendales: Psicóloga Universidad Pedagógica y Tecnológica de Colombia. César Armando Rey Anacona: Psicólogo Universidad Nacional de Colombia, Doctor en Psicología Clínica y de la Salud Universidad de Salamanca, profesor de la Universidad Pedagógica y Tecnológica de Colombia.E-mail: cesar.rey@uptc.edu.co.

\section{A B S T R ACT}

This study examined the personality, psychopathological, and sociodemographic characteristics of women that concluded a violent partner relationship $(n=54)$, comparing them with women that continued with this type of relationship $(n=41)$. The participants are between $18-60$ years $(M=37.4)$. The applied instruments were a socio-demographic survey, the Big Five Questionnaire of Personality (Caprara, Barbaranelli, Borgogni \& Perugini, 1995), and the Mini International Neuropsychiatric Interview (Sheehan \& Lecrubier, 2000). The participants that finished the abusive relationship presented bigger punctuations in the dimensions of Agreeableness, Emotional Stability, and Openness, a lower number of children, bigger help-seeking in government institutions after the violence episodes, and a lower prevalence of mayor depressive episode, dysthymia, low suicidal risk, panic disorder, agoraphobia, and generalized anxiety.

Key words authors:

Partner violence, women, personality, psychopathology, demographics.

Key words plus:

Clinical psychology, Big Five Questionnaire (BFQ), descriptive design. 
La violencia de pareja es definida como "cualquier comportamiento dentro de una relación íntima que causa daño físico, psíquico o sexual a los miembros de la relación" (Krug, Dahlberg, Mercy, Zwi \& Lozano, 2003, p. 97) y alcanza, según la misma fuente, entre el 10 y el $69 \%$ de las mujeres, de acuerdo con encuestas poblacionales realizadas en todo el mundo. En Colombia, según la Encuesta Nacional de Salud y Demografía (Asociación Probienestar de la Familia Colombiana [Profamilia], 2005), cada cinco mujeres que viven o han vivido en pareja han sido víctimas de agresiones físicas por parte de su compañero, pero solo un $22 \%$ de ellas denuncia esta situación, cifra similar a la estimada por el Instituto Nacional de Medicina Legal y Ciencias Forenses (2008), según el cual solamente el $20 \%$ de los casos son denunciados ante las autoridades.

Las investigaciones que se han realizado sobre la violencia hacia la mujer por parte de su pareja, se han enfocado en aspectos como el perfil de la mujer maltratada, las características psicológicas del agresor y los efectos del maltrato sobre la salud. Sin embargo, pocas se han centrado en las características de las mujeres que dejan las relaciones de maltrato, las cuales podrían dar luces sobre los factores y las circunstancias que podrían llevar a una mujer a no continuar con la relación, previniendo así muchos de sus efectos, que incluyen problemas de salud mental como el trastorno por estrés postraumático (Echeburúa, Amor \& de Corral, 2002), los síntomas depresivos (Patró \& Limañana, 2005) y un mayor riesgo de suicidio (Amor, Echeburúa, de Corral, Zubizarreta \& Sarasua, 2002; Mertin \& Mohr, 2001), así como problemas emocionales y de comportamiento en los niños y niñas que presencian este tipo de violencia (Holt, Buckley $\&$ Whelan, 2008) y lesiones temporales o permanentes e incluso la muerte (Krug et al., 2003).

Los estudios sobre los factores que inciden en la continuación de la relación violenta por parte de las mujeres, señalan que la dependencia económica y emocional, la existencia de hijos, las creencias de tipo religioso y sociocultural que propenden por mantener la relación conyugal, el miedo, la baja autoestima, los trastornos o síntomas de ansiedad o depresión y la observación y/o haber sido objeto de violencia en la familia de origen, caracterizan a las mujeres que permanecen en este tipo de relaciones, a pesar de los malos tratos (Chantler, 2006; Echeburúa et al., 2002; Griffing et al., 2005; Martín, Aparicio, Salamanca \& Sánchez, 2008). Por su parte, la investigación ha mostrado que entre los factores que pueden llevar a una mujer maltratada a decidir abandonar a su pareja, se encuentra el maltrato excesivo (Erickson \& Drenovsky, 2005) y el apoyo social percibido (Matud, Aguilera, Marrero, Moraza \& Caballeira, 2003). En ese sentido, Campbell, Rose, Kub y Nedd (1998) señalan que el contar con apoyo familiar y social, disponer de cierta autonomía económica y emocional, que los hijos no sean muy pequeños y tener acceso a los recursos comunitarios de apoyo económico, jurídico o psicológico podrían influir en la decisión de dejar la relación maltratante.

Ospina, Jaramillo, Uribe y Cabarcas (2006) realizaron entrevistas con mujeres que rompieron una relación violenta, encontrando que estas vivían un proceso de cambio que consta de cuatro etapas. La primera, el no tener en cuenta, caracterizada por el hecho de que las mujeres asumían la violencia como algo que formaba parte de su vida cotidiana, no dimensionaban la gravedad del maltrato, tenían la esperanza de poder cambiar al maltratador y estaban convencidas de que su deber era permanecer a su lado. La segunda, el darse cuenta, donde las mujeres empezaban a ser conscientes de su situación y a romper con las ataduras de la etapa anterior, flexibilizando su escala de valores frente a su papel como esposas. La tercera etapa, el encontrarse a sí misma, en la cual las mujeres entendían que eran ellas quienes debían romper con la situación de maltrato, empezar a alejarse del agresor y buscar opciones para salir de la situación. Finalmente, el salir adelante sola, donde las mujeres se sentían listas para desistir y capaces de asumir los roles de padre y madre en la crianza de los hijos y dispuestas a correr todos los riesgos que esta decisión conllevaba.

Los resultados de estas investigaciones señalan que existen rasgos psicológicos, como la autonomía y una buena estima, y factores sociodemográficos, como el número de hijos, que pueden llevar a una mujer a romper con la relación de maltrato, así 
como la ausencia de síntomas y trastornos emocionales, como el episodio depresivo mayor y el trastorno por estrés postraumático. Con base en estas consideraciones, se puede plantear la siguiente pregunta de investigación: ¿Qué características de personalidad, psicopatológicas y sociodemográficas presenta un grupo de mujeres que han dejado una relación de pareja maltratante? Para responderla, este estudio tuvo como objetivo caracterizar a un grupo de mujeres que dejaron este tipo de relación, con base en un conjunto de variables de personalidad, psicopatológicas y sociodemográficas, comparándolas con mujeres con similar edad, nivel educativo y económico, que continuaban con esa misma relación. Para ello y a la luz de los estudios previos, se examinaron las siguientes características sociodemográficas: a) el número de hijos, b) la edad promedio de los hijos, c) el tiempo transcurrido entre el inicio de la relación y el primer episodio de violencia, d) la ocupación, e) el tipo de apoyo social que buscaban las participantes después de un episodio de violencia y $\mathrm{f}$ ) el atestiguamiento de violencia entre los padres. Los rasgos de personalidad examinados fueron las dimensiones y subdimensiones contempladas en el Big Five Questionnaire (Caprara, Barbaranelli, Borgogni \& Perugini, 1995) y los trastornos psicopatológicos fueron los incluidos en la Mini International Neuropsychiatric Interview (Sheehan \& Lecrubier, 2000).

\section{Método}

\section{Diseño}

Se utilizó un diseño de casos y controles, cuya naturaleza es no experimental, transversal, descriptiva y comparativa (Méndez \& Nahimira, 2000).

\section{Participantes}

La muestra la constituyeron 95 participantes, 80 de ellas usuarias de las Comisarías de Familia de la ciudad de Tunja (Departamento de Boyacá, Colombia) que denunciaron maltrato por parte de su pareja y 15 mujeres que fueron a consulta psicológica por violencia de pareja en una institu- ción de capacitación laboral de la misma ciudad, seleccionadas mediante un muestreo incidental (no probabilístico), con edades comprendidas entre 18 y 60 años $(M=37.40 ; D E=8.76)$. La mayoría eran empleadas, con un nivel educativo incompleto de secundaria y estrato socioeconómico bajo. Las participantes fueron distribuidas en dos grupos de acuerdo al informe de finalización o continuación de la relación de maltrato, referida en una ficha sociodemográfica.

Las 54 participantes que finalizaron esta relación (GF), lo habían hecho hacía nueve meses o más, período de tiempo que se utilizó como criterio de selección, ya que en un estudio longitudinal realizado por Bell, Goodman y Dutton (2007) se concluyó que transcurridos más de nueve meses era difícil que la mujer maltratada restableciera la relación violenta. Estas mujeres tenían edades entre 22 y 60 años $(M=37.83 ; D E=9.003)$. De este grupo, un $27.8 \%$ era de estrato bajo bajo, el $61.1 \%$ de estrato bajo y el $11.1 \%$ de estrato medio bajo. Un $55.6 \%$ correspondía a empleadas y un $20.4 \%$ a trabajadoras independientes; el $44 \%$ tenía estudios de secundaria incompleta y secundaria completa. Las 41 mujeres que continuaron la relación maltratante (GC), por su parte, tenían edades entre 18 y 55 años $(M=36.83 ; D E=8.511)$. El $39.0 \%$ era de estrato bajo bajo, el $48.8 \%$ de estrato bajo y el $12.2 \%$ de estrato medio bajo. Un $43.9 \%$ estaba constituido por empleadas y un $14.6 \%$ por trabajadoras independientes; el 37 \% tenía estudios de secundaria incompleta. Los dos grupos de participantes no diferían, desde el punto de vista estadístico, en cuanto a su promedio de edades, $F(1,94)=0.304, p=0.583)$, estrato socioeconómico, $U(95)=1005, p=0.389$ y nivel educativo, $U(95)=885.5, p=0.087)$.

\section{Instrumentos}

\section{Ficha sociodemográfica}

Se desarrolló en esta investigación con el fin de recoger información sobre las siguientes variables: a) el número de hijos, b) la edad promedio de los hijos, c) el tiempo transcurrido entre el inicio de 
la relación y el primer episodio de violencia, d) la ocupación (empleada, desempleada, estudiante, ama de casa, independiente o pensionada) y e) el tipo de apoyo social que buscaban las participantes después de un episodio de violencia: familiares, amigos, instituciones gubernamentales (por ejemplo, Comisaría de Familia, Centro de Atención a la Violencia Intrafamiliar, estación de Policía), personas de la comunidad (por ejemplo, sacerdote, líder comunitario), profesional particular (por ejemplo, psicólogo, trabajador social) o ninguna persona.

Cuestionario de informe sobre incidentes de violencia conyugal (Rey-Anacona, 2008)

Este instrumento permite informar la presencia de 15 formas de violencia de pareja realizadas tanto por parte del padre hacia la madre como viceversa (30 en total), por medio de preguntas de respuestas "sí-no", la mayoría de las cuales contienen preguntas abiertas que permiten ampliar la información sobre los tipos de violencia presenciados. Los tipos de violencia incluidos en este cuestionario son: violencia física, violencia verbal, amenazas verbales, amenazas con un objeto contundente, confinamiento de la persona en la casa o en un cuarto, prohibiciones, expulsión de la casa a la otra persona, violencia sexual, violencia económica y amenazas a personas cercanas. El cuestionario también permite reportar otras formas de violencia ejercidas por parte del padre hacia la madre, y viceversa. Para su desarrollo, este instrumento fue validado a nivel metodológico por dos jueces, y a nivel de contenido, por tres jueces expertos en violencia en la familia, mostrando un alfa de Cronbach de 0.85 (Rey-Anacona, 2008).

\section{Big Five Questionnaire ([BFQ],} Caprara et al., 1995)

Este instrumento contiene 132 ítems tipo Likert y permite medir las cinco dimensiones fundamentales de la personalidad humana:

1. Energía: inherente a una visión confiada y entusiasta de múltiples aspectos de la vida y compuesta por dos subdimensiones: a) Dinamismo, que refiere comportamientos relacionados con la energía comportamental y b) Dominancia, capacidad para imponerse y sobresalir.

2. Afabilidad: preocupación de tipo altruista y de apoyo emocional a los demás; compuesta por: a) Cooperación/Empatía: capacidad para escuchar necesidades y problemas de los demás y b) Cordialidad/Amabilidad: aspectos relacionados con la afabilidad, confianza y apertura hacia los demás.

3. Tesón: propia de un comportamiento de tipo perseverante, escrupuloso y responsable; la conforman: a) Escrupulosidad, asociada con la fiabilidad, meticulosidad y afán por el orden y b) Perseverancia, que incluye aspectos referidos a la persistencia y a la tenacidad.

4. Estabilidad emocional: abarca la capacidad para afrontar los efectos negativos de la ansiedad, la depresión o la irritabilidad; compuesta por: a) Control de Emociones, es decir, de los estados de tensión ante una situación percibida como emotiva y b) Control de Impulsos: control del propio comportamiento incluso en situaciones de peligro, conflicto o incomodidad.

5. Apertura Mental: sobre todo de tipo intelectual, ante nuevas ideas, valores, sentimientos e intereses; compuesta por: a) Apertura a la Cultura: interés por mantenerse informado, hacia la lectura y hacia la adquisición de nuevos conocimientos y b) Apertura a la Experiencia: capacidad de abrirse a perspectivas diversas y a valores, estilos y modos de vida.

En esta investigación se evaluó la consistencia interna del instrumento por medio de alfa de Cronbach, mostrando un buen nivel de confiabilidad (0.76).

\section{Mini International Neuropsychiatric Interview ([MINI], Sheehan Eु Lecrubier, 2000)}

Es una entrevista estructurada validada para su uso internacional, que permite el diagnóstico de los principales trastornos psiquiátricos del Eje I de la cuarta versión con texto revisado del Manual Diagnóstico y Estadístico de los Trastornos Mentales (Asociación Psiquiátrica Americana [APA], 
2002) y la décima versión de la Clasificación Internacional de Enfermedades (Organización Mundial de la Salud, 1992). Su administración es individual en un período de tiempo breve (en promedio 15 minutos). Los resultados de estudios de validez y de confiabilidad demuestran que la MINI tiene índices de validez y confiabilidad altos (Bobes, 1998; Sheehan et al., 1999).

La MINI evalúa el cumplimiento de los criterios diagnósticos de los siguientes trastornos mentales: Episodio Depresivo Mayor (actual, pasado y con síntomas melancólicos), Trastorno Distímico, Riesgo de Suicidio (leve, moderado y alto), Episodio Maníaco (actual y pasado), Episodio Hipomaníaco (actual y pasado), Trastorno de Angustia (actual, alguna vez en la vida y crisis con síntomas limitados), Agorafobia, Fobia Social, Trastorno Obsesivo Compulsivo, Obsesiones, Compulsiones, Estado por Estrés Postraumático, Trastorno de Ansiedad Generalizada, Dependencia y Abuso de Sustancias Alcohólicas, Dependencia y Abuso de Sustancias no Alcohólicas, Trastornos Psicóticos, Trastorno del Estado de Ánimo con Síntomas Psicóticos, Anorexia Nerviosa, Bulimia Nerviosa y Trastorno Antisocial de la Personalidad.

\section{Procedimiento}

Previo permiso, se revisaron los registros institucionales de los casos vinculados a las diferentes Comisarías de Familia de la ciudad y del servicio de psicología de una institución de capacitación laboral, para obtener un listado de posibles participantes. Debido a que el nivel de riesgo por la participación en este proyecto de investigación era mínimo, según los criterios de la Resolución 008430 de 1993 del Ministerio de Salud, se invitó vía telefónica a las posibles participantes, solicitándoles su consentimiento informado verbal y comunicándoles el objetivo, el procedimiento y el manejo confidencial y anonimato de la información. Una vez obtenido el consentimiento informado, se acordaba una cita con las participantes, donde se administraba de forma grupal la ficha sociodemográfica y el BFQ, se leía y explicaba cada uno de los ítems, debido al bajo nivel educativo de varias de las participantes, mientras que por la naturaleza del instrumento, la MINI se administró individualmente.

Los datos obtenidos se analizaron por medio del paquete estadístico SPSS (Versión 12.0), comparándose a los dos grupos de participantes en las variables nominales por medio de la prueba chi cuadrado (ocupación, tipo de apoyo social, presencia de violencia entre los padres y trastornos psicopatológicos) y las variables de intervalo y de razón mediante el ANOVA de un factor (número de hijos, promedio de edad de los hijos, tiempo transcurrido entre el inicio de la relación y el primer episodio de violencia y dimensiones de personalidad).

\section{Resultados}

En primera instancia se presentan los resultados obtenidos con las variables sociodemográficas, iniciando con las variables de razón y después las nominales; luego, los concernientes a las dimensiones de personalidad y, finalmente, los pertenecientes a las variables psicopatológicas.

Los resultados muestran que los dos grupos de mujeres no diferían con respecto al promedio de edad de los hijos y el tiempo transcurrido entre el inicio de la relación y el primer episodio de violencia, aunque sí en cuanto al número de hijos (véase Tabla 1), ya que el GC mostró un promedio de 3.20 hijos, mientras que el GF informó una media de 2.44 hijos, $F(95.1)=4.630, p=0.034$.

En lo concerniente a la ocupación, no se encontraron diferencias estadísticamente significativas en los porcentajes de los dos grupos de participantes, que informaron cada una de las seis categorías de ocupación examinadas, mientras que con respecto al tipo de apoyo social buscado después de un episodio de violencia, se encontró que el porcentaje de participantes del GF que tendía a buscar apoyo de instituciones gubernamentales (por ejemplo, Comisaría de Familia, Centro de Atención a la Violencia Intrafamiliar, estación de Policía), era significativamente mayor al del otro grupo, $\chi^{2}(95.1)=15.898, p<0.01$, en tanto que este último grupo informó, en un 
TABLA 1

Diferencias entre los grupos en las variables sociodemográficas

\begin{tabular}{|c|c|c|c|c|c|c|}
\hline \multirow{2}{*}{ Variable } & \multicolumn{2}{|c|}{ GF } & \multicolumn{2}{|c|}{ GC } & \multirow{2}{*}{$F$} & \multirow{2}{*}{$p$} \\
\hline & $M$ & $\mathrm{DE}$ & $M$ & $D E$ & & \\
\hline Número de hijos & 2.44 & 1.269 & 3.2 & 2.112 & 4.63 & 0.034 \\
\hline Promedio edad hijos & 12.98 & 7.836 & 12.07 & 7.081 & 0.336 & 0.563 \\
\hline $\begin{array}{l}\text { Tiempo transcurrido } \\
\text { inicio de relación y primer } \\
\text { episodio violencia }\end{array}$ & 15.76 & 15.470 & 18.02 & 16.704 & 0.466 & 0.496 \\
\hline \multirow{2}{*}{ Ocupación } & \multicolumn{4}{|c|}{ Frecuencia } & $\chi^{2}$ & $\mathrm{p}$ \\
\hline & & & GF & GC & & \\
\hline Empleada & & & $30(55.6 \%)$ & $18(43.9 \%)$ & 1.266 & 0.179 \\
\hline Desempleada & & & $4(7.4 \%)$ & $6(14.6 \%)$ & 1.292 & 0.211 \\
\hline Estudiante & & & $3(5.6 \%)$ & $3(7.3 \%)$ & 0.122 & 0.523 \\
\hline Ama de casa & & & $6(11.1 \%)$ & $8(19.5 \%)$ & 1.309 & 0.197 \\
\hline Independiente & & & $11(20.4 \%)$ & $5(12.2 \%)$ & 1.112 & 0.22 \\
\hline Pensionada & & & $0(0 \%)$ & $1(2.4 \%)$ & 1.331 & 0.432 \\
\hline \multirow{2}{*}{ Tipo de apoyo social } & \multicolumn{4}{|c|}{ Frecuencia } & $\chi^{2}$ & $p$ \\
\hline & & & GF & GC & & \\
\hline Familia & & & $16(29.6 \%)$ & $7(17.1 \%)$ & 2.003 & 0.227 \\
\hline Amigos & & & $2(3.7 \%)$ & $5(12.2 \%)$ & 2.462 & 0.233 \\
\hline $\begin{array}{l}\text { Instituciones } \\
\text { gubernamentales }\end{array}$ & & & $26(48.1 \%)$ & $4(9.8 \%)$ & 15.898 & 0 \\
\hline Comunidad & & & $1(1.9 \%)$ & $1(2.4 \%)$ & 0.039 & 1 \\
\hline Ningún tipo de ayuda & & & $9(16.7 \%)$ & $24(58.5 \%)$ & 18.022 & 0 \\
\hline \multirow{3}{*}{$\begin{array}{l}\text { Presencia de violencia entre } \\
\text { los padres }\end{array}$} & & & & & $\chi^{2}$ & $p$ \\
\hline & & & GF & GC & & \\
\hline & & & $41(75.9 \%)$ & $29(70.7 \%)$ & 0,324 & 0.368 \\
\hline
\end{tabular}

Nota. Gral. Grupo total; GF: Grupo de participantes que habían finalizado la relación de maltrato; GC: Grupo de participantes que continuaban dicha relación.

Fuente: elaboración propia.

porcentaje significativamente mayor, que no buscaba ningún tipo de ayuda, $\chi^{2}(95.1)=18.022$, $p=0.000$. En relación con la presencia de violencia entre los padres, no se encontró una diferencia estadísticamente significativa entre el porcentaje de participantes de cada grupo que reportó dicha circunstancia (véase Tabla 1).

Los resultados referentes a las dimensiones de personalidad medidas a través del BFQ (Tabla 2), indican que las participantes del GF presentaron un promedio de puntuaciones significativamente mayor en las dimensiones Afabilidad, $F(95.1)=5.766, p=0.018)$, Estabilidad Emocional, $F(95.1)=5.037, p=0.027$ y Apertura Mental, $F(95.1)=6.630, p=0.012$ ), así como en las subdimensiones Cooperación/Empatía, $F(95.1)=4.265, p=0.042$, Control de Emociones, $F(95.1)=13.247, p=0.000$ y Apertura a la Cultura, $F(95.1)=7.092, p=0.009$, comparadas con las participantes del GC.

En cuanto a las variables psicopatológicas ( $\mathrm{Ta}$ bla 3), se encontró que el grupo de mujeres del GC presentó una prevalencia significativamente mayor de los siguientes trastornos, según los criterios diagnósticos contemplados en la MINI: a) Episodio Depresivo Mayor actual, $\chi^{2}(95.1)=$ 25.738, $p=0.000$; b) Episodio Depresivo Mayor pasado $\chi^{2}(95.1)=7.308, p=0.007$; c) Episodio Depresivo Mayor con Síntomas Melancólicos, $\chi^{2}$ $(95.1)=7579, p=0.008 ; d)$ Trastorno Distímico, 
$\chi^{2}(95.1)=8.435, p=0.005 ;$ e) Riesgo Suicida Leve, $\chi^{2}(95.1)=8.394, p=0.004 ;$ f) Episodio Maníaco, $\left.\chi^{2}(95.1)=4.214, p=0.052 ; g\right)$ Trastorno de Angustia, $\left.\chi^{2}(95.1)=6.351, p=0.012 ; \mathrm{h}\right)$ Trastorno de Angustia alguna vez en la vida, $\chi^{2}(95.1)=13.094$, $p=0.000$; i) Agorafobia, $\chi^{2}(95.1)=11.563$, $p=0.001$ y j) Trastorno de Ansiedad Generalizada, $\chi^{2}(95.1)=12.401, p=0.001$. No se encontraron casos de Riesgo Suicida Moderado, Episodio Maniaco (pasado), Episodio Hipomaníaco (actual), Episodio Hipomaníaco (pasado), Trastorno Psicótico, Anorexia Nerviosa y Bulimia Nerviosa.

TABLA 2

Diferencias por grupo en las dimensiones del BFQ

\begin{tabular}{lcccccc}
\hline \multirow{2}{*}{\multicolumn{1}{c}{ Dimensión/ Subdimensión }} & \multicolumn{2}{c}{ GF } & \multicolumn{2}{c}{ GC } & \multirow{2}{*}{ F } & p \\
\cline { 2 - 5 } & M & DE & M & DE & & 0.709 \\
ENERGÍA & 77.77 & 7.348 & 76.2 & 9.693 & 0.702 & 0.402 \\
Dinamismo & 39.59 & 4.533 & 38.56 & 5.753 & 0.956 & 0.331 \\
Dominancia & 38.07 & 4.505 & 37.63 & 5.319 & 0.19 & 0.664 \\
AFABILIDAD & 79.31 & 7.78 & 75.34 & 8.257 & 5.766 & 0.018 \\
Cooperación/Empatía & 41.98 & 4.932 & 39.8 & 5.288 & 4.265 & 0.042 \\
Cordialidad & 37.33 & 4.019 & 35.54 & 4.828 & 3.913 & 0.051 \\
TESÓN & 80.81 & 7.224 & 80.05 & 9.066 & 0.21 & 0.648 \\
Escrupulosidad & 37.19 & 4.597 & 37.68 & 6.536 & 0.19 & 0.664 \\
Perseverancia & 43.57 & 4.346 & 42.37 & 5.953 & 1.308 & 0.256 \\
ESTABILIDAD EMOCIONAL & 67.13 & 8.87 & 63.2 & 7.891 & 5.037 & 0.027 \\
Control de emociones & 37.41 & 5.794 & 33.27 & 5.06 & 13.247 & 0 \\
Control de impulsos & 29.91 & 5.206 & 29.93 & 5.251 & 0 & 0.986 \\
APERTURA MENTAL & 77.7 & 8.803 & 72.93 & 9.155 & 6.630 & 0.012 \\
Apertura a la cultura & 37.02 & 5.826 & 33.66 & 6.425 & 7.092 & 0.009 \\
Apertura a la experiencia & 40.7 & 4.616 & 39.8 & 4.462 & 0.909 & 0.343 \\
\hline
\end{tabular}

Fuente: elaboración propia.

TABLA 3

Diferencias entre los grupos en los trastornos psicopatológicos

\begin{tabular}{lccccc}
\hline \multirow{2}{*}{ Trastorno } & \multicolumn{3}{c}{ Frecuencia } & \multirow{2}{*}{$\chi^{2}$} & \multirow{2}{*}{$p$} \\
\cline { 2 - 4 } & Gral.(\%) & GF $(\%)$ & GC(\%) & & \\
\hline Episodio Depresivo Mayor (actual) & $24(25.3)$ & $3(5.6)$ & $21(51.2)$ & 25.738 & 0 \\
Episodio Depresivo Mayor (pasado) & $22(23.2)$ & $7(13)$ & $15(36)$ & 7.308 & 0.007 \\
Episodio Depresivo Mayor con Síntomas & $11(11.6)$ & $2(3.7)$ & $9(22)$ & 7.579 & 0.007 \\
Melancólicos & $6(6.6)$ & $0(0)$ & $6(14.6)$ & 8.435 & 0.005 \\
Trastorno Distímico & $14(14.7)$ & $3(5.6)$ & $11(26.8)$ & 8.394 & 0.004 \\
Riesgo Suicida Leve & $3(3.2)$ & $0(0)$ & $3(7.3)$ & 4.08 & 0.077 \\
Riesgo Suicida Alto & $6(6.3)$ & $1(1.9)$ & $5(12.2)$ & 4.214 & 0.052 \\
Episodio Maníaco (actual) & $17(17.9)$ & $5(9.3)$ & $12(29.3)$ & 6.351 & 0.012 \\
Trastorno de Angustia & $9(9.5)$ & $0(0)$ & $9(22)$ & 13.094 & 0 \\
Trastorno de Angustia (alguna vez en la vida) & $3(3.2)$ & $2(3.7)$ & $1(2.4)$ & 0.122 & 0.603 \\
Crisis con síntomas limitados & $11(11.6)$ & $1(1.9)$ & $10(24.4)$ & 11.563 & 0.001 \\
Agorafobia & $5(5.3)$ & $1(1.9)$ & $4(9.8)$ & 2.92 & 0.107 \\
Fobia Social & & & &
\end{tabular}




\begin{tabular}{lccccc}
\hline \multirow{2}{*}{ Trastorno } & \multicolumn{3}{c}{ Frecuencia } & \multirow{2}{*}{$\chi^{2}$} & \multirow{2}{*}{$p$} \\
\cline { 2 - 4 } & Gral.(\%) & GF(\%) & GC(\%) & & \\
\hline Trastorno Obsesivo Compulsivo & $19(20)$ & $9(16.7)$ & $10(24.4)$ & 0.869 & 0.25 \\
Obsesiones & $9(9.5)$ & $5(9.3)$ & $4(9.8)$ & 0.007 & 0.601 \\
Compulsiones & $10(10.5)$ & $4(7.4)$ & $6(14.6)$ & 1.292 & 0.211 \\
Estado por Estrés Postraumático & $4(4.2)$ & $1(1.9)$ & $3(7.3)$ & 1.726 & 0.213 \\
Dependencia y Abuso de Alcohol & $2(2.1)$ & $1(1.9)$ & $1(2.4)$ & 0.039 & 0.68 \\
Dependencia y Abuso de Sustancias no & $3(3.2)$ & $1(1.9)$ & $2(4.9)$ & 0.698 & 0.397 \\
Alcohólicas & $19(20)$ & $4(7.4)$ & $15(36.6)$ & 12.401 & 0.001 \\
Trastorno de Ansiedad Generalizada & $4(4.2)$ & $2(3.7)$ & $2(4.9)$ & 0.08 & 0.581 \\
Trastorno Antisocial de la Personalidad (alguna & & & & & \\
vez en la vida) & &
\end{tabular}

Fuente: elaboración propia.

\section{Discusión}

El objetivo de esta investigación fue caracterizar a nivel de personalidad, psicopatología y rasgos sociodemográficos, a un grupo de mujeres que finalizaron una relación maltratante de pareja, comparándolas con mujeres con similar edad, nivel educativo y económico, que continuaban con esa relación. Los resultados obtenidos a nivel sociodemográfico muestran que un número más bajo de hijos puede influir en la decisión de finalizar la relación por parte de las mujeres maltratadas, mientras que lo contrario influiría en el hecho de continuar con dicha relación, algo señalado previamente por algunos autores (Klevens, 2001; Fontanil et al., 2005). También indican que ni el estado ocupacional ni el promedio de edad de los hijos caracterizan a las mujeres que finalizan o continúan con este tipo de relaciones, lo cual es contrario a la consideración de que la independencia económica y la ausencia de hijos pequeños podrían incidir en la decisión de finalizar la relación (Campbell et al., 1998).

Asimismo, los resultados indican que las mujeres que deciden dejar este tipo de relaciones tienden a buscar algún tipo de ayuda después de los incidentes de violencia, en particular, de instituciones gubernamentales, mientras que las mujeres que continúan con la relación no, confirmando que la percepción de contar con apoyo social podría influir en la decisión de finalizar las relaciones de maltrato (Campbell et al., 1998; Matud et al., 2003). A la luz del modelo de cuatro etapas descrito por
Ospina et al. (2006), estos resultados confirman que las mujeres que continúan en la relación tienden a encontrarse en una etapa de no tener en cuenta, que se caracteriza por considerar los malos tratos como algo posible y aceptable en la relación, con el fin de mantener la estabilidad familiar, por lo que tenderían a no buscar ayuda. Por el contrario, las mujeres que deciden dejar la relación se encontrarían en la etapa de encontrarse a sí misma, que se caracteriza por buscar opciones para salir de esta situación y que es posterior a la etapa de darse cuenta, donde modificarían su escala de valores debido a una concientización sobre su situación personal y familiar (Ospina et al., 2006).

Los resultados también señalan que el atestiguamiento de violencia entre los padres no incide en la decisión de finalizar la relación maltratante, aunque confirma los resultados de las investigaciones que muestran que un número importante de las víctimas de violencia de pareja observaron este tipo de violencia entre sus padres (e. g., Fernández-Montalvo \& Echeburúa, 1997; Klevens, 2001; Langhinrichsen-Rohling, Neidig \& Thorn, 1995; Matud et al., 2003; Rey, 2002; Rubiano, Hernández, Molina, Gutiérrez \& Vejarano, 2003), ya que el $73.7 \%$ de toda la muestra informó alguna forma de violencia entre los padres, siendo este porcentajes similar entre los dos grupos de participantes. Estos resultados indican que efectivamente la observación de violencia en la familia de origen puede favorecer los malos tratos, en la medida en que los normalizan y legitiman en la relación, y que 
otros factores serían los responsables de la decisión de dejar la relación de maltrato, como los anteriormente descritos.

Respecto a las dimensiones de personalidad, los resultados muestran, en ese sentido, que las participantes del grupo que finalizó la relación, presentaban mayores puntuaciones en Afabilidad, caracterizada por empatía, apertura hacia otras personas, comprensión de sus problemas y de las necesidades de otros, en Estabilidad Emocional, indicando mayor control de sus experiencias emocionales y capacidad de controlar respuestas a situaciones conflictivas y en Apertura Mental, la cual se asocia con una mejor disposición hacia las experiencias nuevas, ideas, valores y acciones, sin mucho temor al cambio (Caprara et al., 1995). Asimismo, presentaron mayores puntuaciones en las subdimensiones Cooperación/Empatía, indicando una mayor tendencia a escuchar las necesidades y los problemas de los demás, así como a establecer una cooperación con ellos, Control de Emociones, lo que señala un mayor control de los estados de tensión ante una situación percibida como emotiva y Apertura a la Cultura, señalando un mayor interés por mantenerse informado, hacia la lectura y hacia la adquisición de nuevos conocimientos.

Evidentemente, este tipo de rasgos podrían favorecer la decisión de no continuar con la relación, al promover la búsqueda de nuevas alternativas para la situación de maltrato, con una menor experiencia de ansiedad y mayor apertura mental (Chantler, 2006; Fajardo, Fernández \& Escobar, 2002; Martín et al., 2008; Matud et al., 2003; Roberts, 2006). Conforme al modelo de cuatro etapas descrito por Ospina et al. (2006), rasgos como estos podrían llevar a una mujer maltratada a salir adelante sola, ya que la afabilidad y la apertura mental la llevarían a ser conciente de que debe salir adelante en pro de su bienestar y el de sus hijos, y plantearse metas a mediano y largo plazo sin compañía. Desde un enfoque de costes y beneficios, esta apertura mental podría llevar a la mujer maltratada a vislumbrar más beneficios que costes por dicha finalización, llevándola a tomar esta decisión (Amor, Bohórquez \& Echeburúa, 2006).
Por el contrario, las menores puntuaciones en Afabilidad, Estabilidad Emocional, Apertura Mental, Cooperación/Empatía, Control de Emociones y Apertura a la Cultura, entre las mujeres que continuaban la relación, confirma que una personalidad caracterizada por el temor a vivir nuevas experiencias, una mayor tendencia a responder con ansiedad ante las situaciones estresantes de la vida y comprender menos las propias necesidades y las de los demás, influirían negativamente en la decisión de continuar con la relación, ya que estos rasgos corresponden a bajas puntuaciones en estas escalas (Caprara et al., 1995).

A nivel psicopatológico, los resultados muestran que las mujeres que finalizan la relación tienen una más baja probabilidad de presentar episodio depresivo mayor (pasado, actual o con síntomas de melancolía), así como riesgo suicida leve y distimia, a diferencia de las mujeres que continúan con la relación. Estos resultados indican que la presencia de un trastorno depresivo o de distimia limita significativamente la decisión de finalizar una relación de maltrato, lo cual es comprensible si se tiene en cuenta que este tipo de trastornos afectan negativamente las áreas afectiva, motivacional y cognoscitiva del individuo, con síntomas como dificultades para tomar decisiones, estado de ánimo triste, baja autoestima, poca energía, visión negativa de sí mismo y sentimientos de desesperanza (APA, 2002). Uno de los correlatos del episodio depresivo mayor lo constituyen las ideas suicidas, como efectivamente se encontró en esta investigación al hallarse una mayor prevalencia de riesgo suicida leve entre las mujeres que continuaron la relación maltratante.

Estos hallazgos son coherentes con la explicación de los síntomas de la violencia hacia la mujer que se ha realizado desde la teoría de la indefensión aprendida, según la cual cuando una persona, durante un periodo prolongado de tiempo, sufre maltrato de forma impredecible e incontrolable, llega a un estado de indefensión que conduce a síntomas depresivos como los descritos, haciendo más probable que continúe en la relación (Amor et al., 2006). Efectivamente, los resultados muestran que las mujeres maltratadas pueden estar más propensas a los trastornos depresivos y la distimia, probable- 
mente como efecto de la situación de maltrato, y a una percepción de amenaza continua y sin salida de dicha situación.

Desde el punto de vista psicopatológico, también se encontró que las participantes del grupo que continuaron la relación presentaban una mayor prevalencia de tres trastornos de ansiedad (v. g., Trastorno de Angustia, Agorafobia y Trastorno de Ansiedad Generalizada). El trastorno de angustia con o sin agorafobia, es altamente incapacitante, pues puede llevar al individuo a permanecer encerrado en su casa, limitando sus posibilidades de desarrollo personal y social (APA, 2002). Si bien los resultados obtenidos no permiten determinar si estos trastornos fueron favorecidos por los malos tratos, sus síntomas explicarían claramente por qué este grupo de mujeres tendería a continuar la relación. El trastorno de ansiedad generalizada, por su parte, se caracteriza por la constante preocupación y miedo que interfieren en diferentes aspectos de la vida, lo que explicaría su más alta prevalencia dentro del grupo que continuó, ya que esta condición limitaría la posibilidad de romper la relación, porque la mujer se sentiría más segura dentro de ella, evitando el miedo a afrontar las situaciones a las que teme constantemente.

Según todo lo anterior, un perfil de mujeres que finalizan una relación maltratante podría ser el siguiente: mujeres con dos hijos o menos que buscan ayuda en instituciones gubernamentales, que presentan características de personalidad de tipo altruista y comprensión de los problemas y mayor control de las emociones, junto con una mayor capacidad de dar respuesta a situaciones de conflicto y peligro, además de tener flexibilidad de pensamiento y disposición a experiencias nuevas. Por último, no presentarían trastornos ansiosos y depresivos o sintomatología ansiosa y depresiva clínicamente significativa.

Esta investigación tuvo como principales limitaciones el tamaño y la selección de la muestra, ya que esta fue incidental y subordinada a la voluntariedad y disponibilidad de tiempo de las participantes, realizándose el reclutamiento solamente en algunas instituciones de la ciudad. Además, en general las participantes tenían un nivel educativo y socioeco- nómico bajo, lo cual limita la generalización de los resultados, y su rango de edad era tan amplio que ameritaba un análisis por diferentes edades, el cual no se realizó. Otra limitación está relacionada con el uso del BFQ, ya que no se cuenta con datos de confiabilidad y validez obtenidos con muestras colombianas. Aún así, la consistencia interna del instrumento fue de 0.766 , lo que confirma que su confiabilidad es altamente satisfactoria.

\section{Referencias}

Amor, P., Echeburúa, E., de Corral, P., Zubizarreta, I. \& Sarasua, B. (2001). Repercusiones psicopatológicas de la violencia doméstica en la mujer en función de las circunstancias de maltrato. Revista Internacional de Psicología Clínica y de la Salud, 2(2), 227-242.

Amor, P., Bohórquez, I. A. \& Echeburúa, E. (2006). iPor qué y a qué coste físico y psicológico permanece la mujer junto a su pareja maltratadora? Acción Psicológica, 4(2), 129-154.

Asociación Probienestar de la Familia Colombiana. (2005). Encuesta Nacional de Salud y Demografía 2005. Recuperado el 17 de julio de 2006, de httpwww.profamilia.org.co/encuestas01encuestas2005resultados_generales.htm

Asociación Psiquiátrica Americana. (2002). Manual Diagnóstico y Estadístico de los Trastornos Mentales (4⿳亠丷厂 ed. rev.). Madrid: Masson.

Bell, M., Goodman L. \& Dutton, M. (2007). The dynamics of staying and leaving: Implications for battered women's emotional well-being and experiences of violence at the end of a year. Journal Family Violence, 22(6), 413-428.

Bobes, J. (1998). A Spanish validation study of the Mini International Neuropsychiatric Interview. European Psychiatry, 13(4), 198-199.

Campbell, J., Rose, L., Kub, J. \& Nedd, D. (1998). Voices of strength and resistance: A contextual and longitudinal analysis of women's responses to battering. Journal of Interpersonal Violence, 13(6), 743-762.

Caprara, G. V., Barbaranelli, C., Borgogni, L. \& Perugini, M. (1995). BFQ Cuestionario "Big Five". Madrid: TEA.

Chantler, K. (2006). Independence, dependency, and interdependence: Struggles and resistances of 
minoritized women within and on leaving violent relationships. Feminist Review, 82(1), 27-49.

Echeburúa, E., Amor, J. \& de Corral, P. (2002). Mujeres maltratadas en convivencia prolongada con el agresor: variables relevantes. Acción Psicológica, 2(7), 135-150.

Erickson, R. \& Drenovsky, C. (2005). The decision to leave an abusive relationship: The testing of an alternative methodological approach. Journal of Family Violence, 5(3), 237-246.

Fajardo, M., Fernández, H. \& Escobar, A. (2002, 14 de octubre). Estilo de vida, perfil psicológico y demográfico de mujeres maltratadas por su cónyuge. Revista PsicologíaCientífica.com, 4(12). Recuperado el 28 de julio de 2008, de http://www.psicologiacientifica.com/bv/psicologia-107-1-estilo-de-vida-perfilpsicologico-y-demografico-de-mujeres-m.html

Fernández-Montalvo, J. \& Echeburúa, E. (1997). Variables psicopatológicas y distorsiones cognitivas de los maltratadores en el hogar: un análisis descriptivo. Análisis y Modificación de Conducta, 23, 151-180.

Fontanil, Y., Ezama, E., Hernández, R., Gil, P., Herrero, F. \& Paz, D. (2005). Prevalencia del maltrato de pareja contra las mujeres. Psicothema, 17(1), 90-95.

Griffing, S., Ragin, D., Morrison, S., Sage, R., Madry, L. \& Primm, B. (2005). Reasons for returning to abusive relationships: Effects of prior victimization. Journal of Family Violence, 20(5), 341-348.

Holt, S., Buckley, H. \& Whelan, S. (2008). The impact of exposure to domestic violence on children and young people: A review of the literature. Child Abuse E Neglect, 32, 797-810.

Instituto Colombiano de Medicina Legal y Ciencias Forenses. (2008). Forensis: datos para la vida. Bogotá: Autor.

Klevens, J. (2001). Violencia física contra la mujer en Santa Fe de Bogotá: prevalencia y factores asociados. Revista Panamericana de Salud Pública, 9(2), 78-83.

Krug, E. G., Dahlberg, L. L., Mercy, J. A., Zwi, A. B. \& Lozano, R. (2003). Informe mundial sobre la violencia y la salud. Washington: Organización Mundial de la Salud.

Langhinrichsen-Rohling, J., Neidig, P. \& Thorn, G. (1995). Violent marriages: Gender differences in levels of current violence and past abuse. Journal of Family Violence, 10(2), 159-176.

Martín, J., Aparicio, D., Salamanca, A. \& Sánchez, S. (2008). Los factores que modulan las decisiones de las mujeres maltratadas. NURE Investigación, 32, 32-41.

Matud, M., Aguilera, L., Marrero, R., Moraza, O. \& Caballeira, A. (2003). El apoyo social en la mujer maltratada por su pareja. Revista Internacional de Psicología Clínica y de la Salud, 3(3), 439-459.

Méndez, I. \& Nahimira, G. (2000). El protocolo de investigación: lineamientos para su elaboración y análisis. México: Trillas.

Mertin, P. \& Mohr, P. (2001). A follow-up study of posttraumatic stress disorder, anxiety and depression in Australian victims of domestic violence. Violence Eg Victims, 16(6), 645-654.

Organización Mundial de la Salud. (1992). Décima revisión de la Clasificación Internacional de Enfermedades (CIE-10): trastornos mentales y del comportamiento. Madrid: OMS/Meditor.

Ospina, D., Jaramillo, D., Uribe, T. \& Cabarcas, G. (2006). Escala de identificación de las etapas de cambio conductual en mujeres en una relación conyugal violenta. Revista Latinoamericana de Psicología, 38(3), 523-534.

Patró, R. \& Limiñana R. (2005). Depresión en mujeres maltratadas: relaciones con estilos de personalidad, variables contextuales y de la situación de violencia. Anales de Psicología, 21(1), 11-17.

Resolución 008430 de 1993 [Ministerio de Salud]. Por la cual se establecen las normas científicas, técnicas y administrativas para la investigación en salud. Octubre 4 de 1993.

Rey, C. (2002). Rasgos sociodemográficos e historia de maltrato en la familia de origen, de un grupo de hombres que han ejercido violencia hacia su pareja y de un grupo de mujeres víctimas de este tipo de violencia. Revista Colombiana de Psicología, 11, 81-90.

Rey-Anacona, C. A. (2008). Habilidades pro sociales, rasgos de personalidad de género y aceptación de la violencia hacia la mujer, en adolescentes que han presenciado violencia entre sus padres. Acta Colombiana de Psicología, 11(1), 107-118. 
Roberts, A. (2006). Classification typology and assessment of five levels of woman battering. Journal of Family Violence, 8(21), 521-527.

Rubiano, N., Hernández, A., Molina, C., Gutiérrez, M. \& Vejarano, M. (2003). Conflicto y violencia intrafamiliar. Bogotá: Universidad Externado de Colombia.
Sheehan, D. V. \& Lecrubier, Y. (2000). Mini International Neuropsychiatric Interview: versión en español 5.0.0. Manuscrito no publicado.

Sheehan, D., Lecrubier, Y., Sheehan, H., Janavs, J., Weiller, E., Keskiner, A. et al. (1999). The validity of the Mini International Neuropsychiatric Interview (MINI) according to the SCID-P and its reliability. European Psychiatry, 12(5), 232-241. 